\title{
Effects of coenzyme Q10 on semen cryopreservation of stallions classified as having good or bad semen freezing ability
}

\author{
Joao A.M. Carneiro ${ }^{\mathrm{a}}$, Igor F. Canisso ${ }^{\mathrm{b}}$, R.S. Bandeira ${ }^{\mathrm{a}}$, V.F.C. Scheeren ${ }^{\mathrm{a}}$, \\ Camila P. Freitas-Dell'Aqua ${ }^{a}$, Marco A. Alvarenga ${ }^{a}$, Frederico O. Papa ${ }^{a}$, \\ Jose A. Dell'Aqua Jr., \\ a Sao Paulo State University (UNESP), School of Veterinary Medicine and Animal Science, Botucatu, Brazil \\ ${ }^{\mathrm{b}}$ Department of Veterinary Clinical Medicine, College of Veterinary Medicine, University of Illinois Urbana-Champaign, Urbana, IL, 61802, USA
}

\section{A R T I C L E I N F O}

\section{Keywords:}

Antioxidant

Semen freezing

Horse

Oxidative stress

Cryodamage

\begin{abstract}
A B S T R A C T
This study aimed to evaluate the antioxidant properties of coenzyme Q10 (CoQ10) during cryopreservation of semen obtained from stallions having good and bad semen freezing ability (GFA vs. BFA, respectively). Forty ejaculates ( $\mathrm{n}=20$ stallions) were split into five centrifugation and five freezing extenders containing different concentrations of CoQ10 $(0,25,50,75$ and $100 \mu \mathrm{mols} / \mathrm{L}$ ). If CoQ10 was added to the centrifugation extender, the freezing extender had no CoQ10 added; similarly, if CoQ10 was added to the freezing extender, the centrifugation extender had no CoQ10. Semen cryopreserved on extenders containing no CoQ10 served as the control. After post-thaw total sperm motility (TM) assessments, the stallions were classified as GFA (i.e., decrease of $\leq 25 \%$ in TM, $n=7$ ) or BFA (i.e., decrease of $\geq 40 \%$ in $T M, n=5$ ). Stallions not fitting $(n=8)$ this enrollment criteria had samples discarded. After that, two straws for each extender were thawed at $37^{\circ} \mathrm{C}$ for $30 \mathrm{~s}$; one straw was immediately used for evaluation of sperm kinetics, plasma membrane integrity, non-capacitated spermatozoa, reactive oxygen species production, mitochondrial activity and lipid peroxidation. The second straw was kept at $37^{\circ} \mathrm{C}$ for $30 \mathrm{~min}$ and subjected to the same assessments. Expectedly, sperm motility parameters were significantly lower for stallions with BFA. There were no effects of CoQ10 concentration or time for all parameters evaluated in the group with GFA when compared with the control extender ( $p>0.05$ ), except lipid peroxidation ( $\mathrm{p}<0.05$ ). However, stallions with BFA had improved sperm parameters for samples processed with extenders containing CoQ10 (particularly $75 \mu \mathrm{mols} / \mathrm{L})(\mathrm{p}<0.05)$, except for the reactive oxygen species production and mitochondrial potential (T0) in which there were no differences between the groups ( $\mathrm{p}>0.05$ ). In summary, $75 \mu \mathrm{mols} / \mathrm{L}$ appears to be the optimal dose of Co-Q10, particularly, when added to the centrifugation extender.
\end{abstract}

\section{Introduction}

Semen cryopreservation is the greatest tool to preserve the genetics of a sire for unlimited period and to allow worldwide use of a desired stallion. Unlike other farm animals, the genetic selection of horses is purely based on desired phenotypic features, pedigree, and athletic achievements and rarely based on fertility (Brito, 2007; Moore et al., 2005; Morillo-Rodríguez et al., 2012). Unintentionally, this approach has likely favored the dissemination of sires with poor fertility unable to tolerate the various steps involved

\footnotetext{
* Corresponding author.

E-mail address: dellaquajunior@uol.com.br (J.A. Dell'Aqua).
} 
on standard semen cryopreservation. Therefore, there is a critical need to develop new strategies to improve semen cryopreservation of stallions with poor semen freezing ability.

Invariably semen cryopreservation results in some degree of cryoinjuries to sperm cells such as lipid peroxidation, excessive consumption of intracellular ATP, damage to plasma membrane and acrosome, reduction of mitochondrial membrane potential, and sperm motility (Sieme et al., 2008; Gibb and Aitken, 2016). The different types of cryodamage are interdependent and often a side effect of abnormal production of reactive oxygen species (ROS) (García et al., 2011; Pisoschi and Pop, 2015). Interesting that some stallions appear to be more or less susceptible than others are to cryoinjuries during standard semen cryopreservation. Arbitrarily, stallions have been classified according to their ability to tolerate standard semen cryopreservation process, as having good (i.e., decrease of $\leq 25 \%$ in total sperm motility post-thaw) or bad (i.e., decrease of $\geq 40 \%$ in total sperm motility post-thaw) semen freezing ability (Hartwig et al., 2014).

Studies performed over the years have improved stallion semen cryopreservation (Martin et al., 1979; Squires et al., 2004; Vidament, 2005; Sieme et al., 2008; Papa et al., 2008; Rodríguez et al., 2011). However, some stallions still unable to tolerate standard semen freezing process (Hartwig et al., 2014). It appears that stallions with poor post-thaw sperm motility results after standard semen freezing have a reduced intrinsic antioxidant ability. Of interest, subfertility in man have been associated with uncontrolled oxidative stress (i.e., excessive ROS production) (Gvozdjakova et al., 2015, 2013). In horses, multiple studies have attempted to neutralize the effects of ROS by adding antioxidants (e.g., superoxide dismutase, catalase, glutathione peroxidase, ascorbic acid, $\alpha$-tocopherol, ascorbic acid, quercetin, butylated hydroxytoluene, L-carnitine) to semen extenders with variable results (Andrade et al., 2010; Gibb and Aitken, 2016; Granollers et al., 2011; Lenzi et al., 2002; Luz et al., 2011; Seifi-Jamadi et al., 2016a, b).

Coenzyme Q10 is a redox lipid, ubiquitously expressed in mammalian cells, able to promote energy generation via exchange of electrons and protons in the electron transport chain of internal mitochondrial membrane (Carocho and Ferreira, 2013; Lewin and Lavon, 1997; Patel and Sigman, 2008). It is also a potent antioxidant present in lipoproteins, capable to prevent lipid peroxidation by inhibiting the formation of hydroperoxides (Alleva et al., 1997; Bentinger et al., 2007; Carocho and Ferreira, 2013; Lewin and Lavon, 1997; Patel and Sigman, 2008). Coenzyme Q10 has been shown to improve stallion semen cooling (Nogueira, 2015; Yousefian et al., 2014), and subfertility in man by preventing lipid peroxidation and promoting sperm motility and DNA integrity (Agarwal and Majzoub, 2017; Talevi et al., 2013). To date, the effects of coenzyme Q10 has yet to be assessed for stallion semen cryopreservation.

In light of the present knowledge, we hypothesized that inclusion of coenzyme Q10 to semen extenders improve stallion semen cryopreservation in a dose-depend manner. Since the oxidative stress during cryopreservation is not exclusively generated during freezing and thawing, but also during centrifugation, we assessed the addition of different concentrations of coenzyme Q10 to the extender used for centrifugation and cryopreservation. As stallions classified as having good semen freezing ability typically have satisfactory post-thaw motility and pregnancy rates under good breeding management (Maziero, 2010; Hartwig et al., 2014), we were mostly interested in comparing the addition of coenzyme Q10 to extenders for this type of stallion with those classified as having bad semen freezing ability.

\section{Material and methods}

All the reagents used in the study were purchased from Sigma-Aldrich (St. Louis, MO, USA) unless stated otherwise. The experimental protocols carried out herein were reviewed and approved by the Ethics Committee for Animal Use, São Paulo State University, under protocol \# 89/2016. This study was carried out from May to July of 2016. The animals were housed on a private equine breeding center located in Bauru, São Paulo, Brazil.

\subsection{Stallions, semen collection and processing, and experimental design}

Two ejaculates were collected from twenty stallions (Mangalarga Marchador $n=7$, Quarter Horse $n=7$, and Thoroughbred $\mathrm{n}=6$ ) ranging from 5 to 20 years-old. The animals were kept in stalls with free choice access to hay, water, and trace minerals. Semen collection was performed using a Botucatu artificial vagina (Botupharma, Botucatu, São Paulo, Brazil) off a dummy mount in the presence of a teaser mare. Following the collection, each ejaculate was filtered to remove debris and the gel fraction. On field, sperm motility was subjectively assessed, and gel-free semen volume recorded. After that, sperm concentration was determined with a hemocytometer in standard fashion using a 1:20 dilution with distilled water.

Following determination of concentration, semen was extended to 50 million sperm/mL with a commercial skim milk-based extender (Botu-Semen ${ }^{\circledR}$, Botupharma, Botucatu, São Paulo, Brazil). After that, each ejaculate was equally split into nine subgroups according to the concentrations of coenzyme Q10 added and extender used for centrifugation (CE) or freezing (FR) (Table 1). Groups having coenzyme Q10 added into the centrifugation extender did not have any of this antioxidant added to the freezing extender. Similarly, groups having coenzyme Q10 added to the semen freezing extenders were centrifuged with skim milk-based extender containing no coenzyme Q10 (Table 1). The control had no coenzyme Q10 added to either centrifugation or freezing extenders.

After extension, semen was centrifuged at $600 \mathrm{xg}$ for $10 \mathrm{~min}$, the supernatant discarded and the pellet resuspended in a commercially available, egg-yolk-based semen freezing extender (Botu-Crio ${ }^{\circledR}$, Botupharma, Botucatu, São Paulo, Brazil) added or not of coenzyme Q10 (Table 1). Thereafter, sperm concentration was adjusted to 200 million sperm/mL, loaded in $0.5 \mathrm{~mL}$ straws, placed in a temperature-controlled refrigerator (Minitub do Brasil Ltda, Porto Alegre, Rio Grande do Sul, Brazil) at $5{ }^{\circ} \mathrm{C}$ for 20 min. Afterwards, the straws were placed $6 \mathrm{~cm}$ above liquid nitrogen for $20 \mathrm{~min}$ and then plunged into the liquid nitrogen (Papa et al., 2008). Three weeks after semen freezing, samples were transported from the breeding center to the investigators' laboratory for further semen 
Table 1

Group codes for the different extenders according to coenzyme Q10 concentrations, the control group consisted of the original centrifugation and freezing extenders.

\begin{tabular}{lll}
\hline Concentrations of coenzyme Q10 & \multicolumn{2}{c}{ Extenders (Code) } \\
\cline { 2 - 3 } & Centrifugation (CE) & Freezing (FR) \\
\hline $0 \mu \mathrm{mol} / \mathrm{L}$ & Control & Control \\
$25 \mu \mathrm{mol} / \mathrm{L}$ & CE25 & FR25 \\
$50 \mu \mathrm{mol} / \mathrm{L}$ & CE50 & FR50 \\
$75 \mu \mathrm{mol} / \mathrm{L}$ & CE75 & FR75 \\
$100 \mu \mathrm{mol} / \mathrm{L}$ & CE100 & FR100 \\
\hline
\end{tabular}

analyses. Two straws were thawed at $37^{\circ} \mathrm{C}$ for $30 \mathrm{~s}$ in a water bath. Immediately after thawing, the content of one straw was transferred to $1.5 \mathrm{~mL}$ and kept at $37^{\circ} \mathrm{C}$ for immediate sperm analyses (T0) as described below, whereas, the remaining straw was kept sealed in the water bath for $30 \mathrm{~min}$ (T30) at $37^{\circ} \mathrm{C}$ for similar sperm assessments.

\subsection{Animals classification based on semen freezing ability}

Immediately after thawing, semen samples $(n=40)$ from all twenty stallions were evaluated for total sperm motility as described below. Based on post-thaw motility results of both ejaculates, stallions were classified as having good (i.e., decrease of $\leq 25 \%$ in total sperm motility) or bad (i.e., decrease of $\geq 40 \%$ in total sperm motility) semen freezing ability (Hartwig et al., 2014). Of the twenty stallions, seven (Quarter Horse $n=5$; Thoroughbred $n=2$ ) were classified as having good semen freezing ability and five (Mangalarga Marchador $n=4$; Quarter Horse $n=1$ ) as having bad semen freezing ability. The remaining eight stallions did not fit the study's inclusion criteria. Thus, their samples were discarded.

\subsection{Sperm motility parameters}

The sperm motility parameters were evaluated with a Computer Assisted-Sperm Analyzer (HTM-IVOS 12, Hamilton Thorne Research, Beverly, MA) by assessing five random fields. For each sample, the percentage of total sperm motility, progressive motility, and fast-moving spermatozoa were evaluated using customized settings for equine sperm (Table 2).

\subsection{Flow cytometry analysis}

Flow cytometric analyses were carried out with Fortessa LSR equipment (Becton Dickinson, Mountain View, CA, USA) equipped with blue (488-nm, $100 \mathrm{~mW})$, red (640-nm, $40 \mathrm{~mW}$ ) and violet (405-m, $100 \mathrm{~mW}$ ) lasers. After the flow cytometric assays, the data were extracted using the manufacturer's software (BD FACSDiva ${ }^{\mathrm{TM}} \mathrm{v6.1}$ ). For flow cytometry assays, all the samples were extended in TALP-PVA containing Hoescht 33,342 (100 $\mu \mathrm{g} / \mathrm{mL}$ ) to discard the non-cellular particles (Parrish et al., 1988). The composition of the

Table 2

Settings for stallion semen analyses.

\begin{tabular}{ll}
\hline Features & Setting \\
\hline Image capture (frames per sec) & $60 \mathrm{~Hz}$ \\
Image capture (No. of frames) & 30 \\
Cell detection (min contrast) & 30 \\
Cell detection (min cell size) & 30 pixels \\
Defaults (cell size) & 5 pixels \\
Defaults (cell intensity) & 40 \\
Progressive cells (VAP) & $70 \mu \mathrm{m} / \mathrm{s}$ \\
Progressive cells (STR) & $80 \%$ \\
Slow cells (static: VAP cutoff) & $30 \mu \mathrm{m} / \mathrm{s}$ \\
Slow cells (static: VSL cutoff) & $20 \mu \mathrm{m} / \mathrm{s}$ \\
Illumination: intensity & 3,600 \\
Illumination: photometer & 125 \\
Video source (dark field) & $60 \mathrm{~Hz}$ \\
Static intensity gates (min and max) & 0.48 and 1.45 \\
Static elongation gates (min and max) & 0 and 97 \\
Chamber-type & Makler ${ }^{1}$ \\
Temperature & $38{ }^{\circ} \mathrm{C}$ \\
Field selection & Automatic \\
\hline
\end{tabular}

VAP average path velocity $\mu \mathrm{m} / \mathrm{s}$, VSL velocity straight line $\mu \mathrm{m} / \mathrm{s}$; STR straight $\%$ ${ }^{1}$ Hamilton Thorne Research, Beverly, MA, USA (chamber depth $10.0 \mu \mathrm{m}$, stage position $14.3 \mathrm{~mm})$. 
TALP-PVA medium was as follows: $100 \mathrm{mM} \mathrm{NaCl}, 3.1 \mathrm{mM} \mathrm{KCl}, 25.0 \mathrm{mM}$ NaHCO 3, $0.3 \mathrm{mM}$ NaH 2 PO 4, $21.6 \mathrm{mM}$ DL 60\% sodium lactate, $2.0 \mathrm{mM} \mathrm{CaCl} 2,0.4 \mathrm{mM} \mathrm{MgCl} 2,10.0 \mathrm{mM}$ Hepes-free acid, $1.0 \mathrm{mM}$ sodium pyruvate, $1.0 \mathrm{mg} / \mathrm{mL}$ polyvinyl alcohol-PVA and $25 \mu \mathrm{g} / \mathrm{mL}$ gentamicin. Auto-fluorescence and controls of each fluorochrome were acquired for adjustment of wave overlap and compensation using the matrix compensation of the flow cytometer software. The data were generated using histogram graphs including axis < 0t (bi-exponential) making all events visible and properly compensated. For each assay, at least 10,000 cells per sample were analyzed.

For the evaluation of intracellular hydrogen peroxide (H2O2) production, dihydrorhodamine 123 (DHR, D23806, Life Technologies, São Paulo, São Paulo, Brazil) was used in combination with propidium iodide. After reacting with ROS such as hydrogen peroxide or peroxide nitrite, dihydrorhodamine 123DHR is oxidized into a fluorescent compound (rhodamine 123), which is retained within the cell.

Each $500 \mu \mathrm{L}$ aliquot of extended semen in TALP-PVA had $1.5 \mu \mathrm{M}$ propidium iodide (diluted in TALP-PVA) and $1 \mu \mathrm{M}$ DHR (diluted in dimethyl sulfoxide) added before being incubated at $37^{\circ} \mathrm{C}$ for $20 \mathrm{~min}$ under the light. Assessments of mitochondrial membrane potential, intracellular superoxide (O2-) production and plasma membrane stability were carried out, with the association of MitoStatusRed (MST, Mitochondrial Potential) and Dihydroethidium (DHE, D23107 - Life Technologies) and Yo-Pro (YP, Y3603 Life Technologies, changes in membrane permeability, through the generation of intracytoplasmic superoxide anion. Thus, YP (25 nM), MST $(20 \mu \mathrm{M})$ and DHE $(2 \mu \mathrm{M})$ were added to the $500 \mu \mathrm{L}$ semen aliquots extended in TALP-PVA and then incubated at $37^{\circ} \mathrm{C}$ for 20 min. Changes in plasma membrane (e.g., capacitation) makes the membrane permeable to small cationic molecules such as the YoPro while remaining impermeable to large cationic molecules such as propidium iodide. The MitoStatus reagent is a lipophilic cation that accumulates inside the mitochondria emitting fluorescence when there is mitochondrial membrane potential. Dihydroethidium is permeable to the plasma membrane and widely used to monitor the production of superoxide. When oxidized by superoxide anions into 2-hydroxyethidium, it forms a red fluorescent product (ethidium) that intercalates with DNA.

Lipid peroxidation assessments were carried out with the fluorescent probe C11-BODYPY (D-3861; Molecular Probes, Carlsbad, CA, USA), is an lipophilic fluorophore sensitive to react with oxygen and peroxynitrite and once oxidized converts its fluorescence from red to green. Each aliquot of semen ( 2 million sperm/mL TALP-PVA extended in $489.5 \mu \mathrm{L}$ ) was added C11BODIPY581/591 $(0.5 \mu \mathrm{L}$, solution $1 \mathrm{mg} / \mathrm{mL})$, propidium iodide $(5 \mu \mathrm{l}$, solution $50 \mu \mathrm{g} / \mathrm{mL})$, Hoescht 33,342 $(5 \mu \mathrm{L}$, solution $100 \mu \mathrm{g} / \mathrm{mL})$ and then the mixture was incubated for at $37^{\circ} \mathrm{C}$ for $30 \mathrm{~min}$. After incubation, the samples were washed two times at $300 \mathrm{~g}$ for $5 \mathrm{~min}$ and the pellet resuspended in $500 \mu \mathrm{L}$ of TALP-PVA and then analyzed by flow cytometry (Guasti et al., 2012).

\subsection{Statistical analysis}

The data analysis was performed with commercial software (Instat 5.0 software (GraphPad Software Inc, USA). The KolmogorovSmirnov test was used to assess the normal distribution of the variables being studied. Since all variables studied herein were not normally distributed, the data analyses was carried out with a non-parametric test of Kruskal-Wallis followed by the Dunn test. Comparisons were performed across groups, times, and concentrations of coenzyme Q10 added. Significance was set at $\mathrm{p}<0.05$. All the data are depicted in tables with variables presented as means \pm SEM to facilitate reader's interpretation.

\section{Results}

As expected, stallions classified as having good semen freezing ability presented significantly better sperm motility parameters than those classified as having bad semen freezing ability at either time-point assessed (Table 3). Addition of coenzyme Q10 improved all motility parameters in comparison to the control group for both time points for stallions with bad semen freezing ability ( $p<0.05$ ), but it did not differ for stallions with good semen freezing ability ( $p>0.05$ ). Overall, addition of coenzyme Q10 to the extender used for centrifugation improved total and progressive sperm motility when compared to the control group. Of interest, $75 \mu \mathrm{mols} / \mathrm{L}$ of coenzyme Q10 appeared to be the most beneficial concentration when added to the centrifugation extender as it maintained the progressive motility after $30 \mathrm{~min}$ of storage at $37{ }^{\circ} \mathrm{C}$ (Table 3 ). It should be noted that while the addition of coenzyme benefited stallions with bad semen freezing ability the sperm kinetics parameters still much inferior to those stallions with good freezing ability $(\mathrm{p}<0.05)$.

Representative histograms for the different flow cytometry parameters are depicted in Figs. 1-3. Membrane integrity and noncapacitated spermatozoa were significantly better for the stallions with good semen freezing ability at both time points ( $\mathrm{p}<0.05$ ), however, there were no significant differences for lipid peroxidation between groups (Table 4). Intracellular superoxide, hydrogen peroxide, and total mitochondrial membrane potential results were not affected by the addition of coenzyme Q10 on either group of stallions or time points (Table 5$)(\mathrm{p}>0.05)$.

\section{Discussion}

This study was carried out to assess whether coenzyme Q10, an antioxidant, could be used to improve stallion semen cryopreservation. To achieve this goal, we tested various concentrations of coenzyme Q10 to the extenders used for centrifugation and cryopreservation. Also, we grouped the stallions as having good and bad semen freezing ability for further analyses. Animals with bad semen freezing ability had sperm motility parameters enhanced by the exposure to coenzyme Q10; this result can probably be explained by the role of coenzyme Q10 in promoting ATP synthesis which is important for sperm motility (Carocho and Ferreira, 2013; Patel and Sigman, 2008; Varner et al., 2014). Our results appear to concur with previous human studies in which the 







\section{Control}
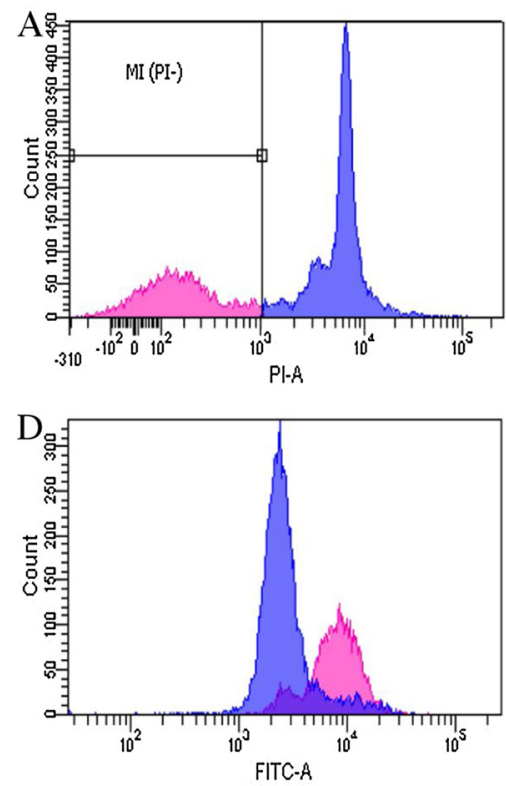

CE75
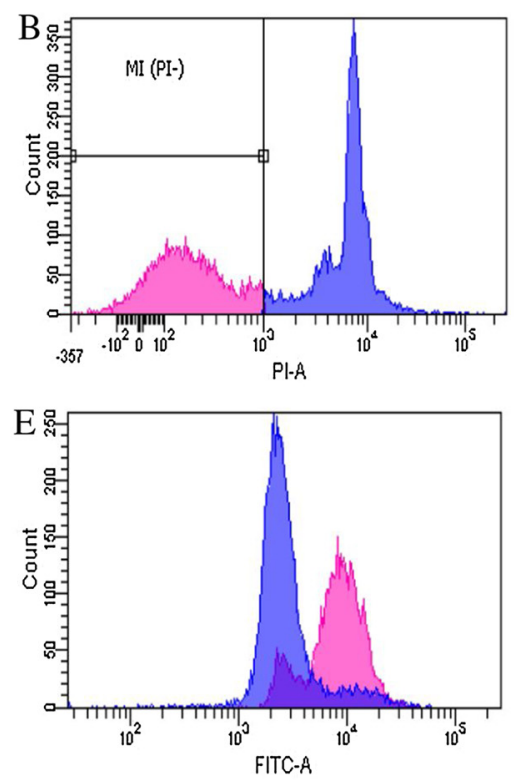

FR100
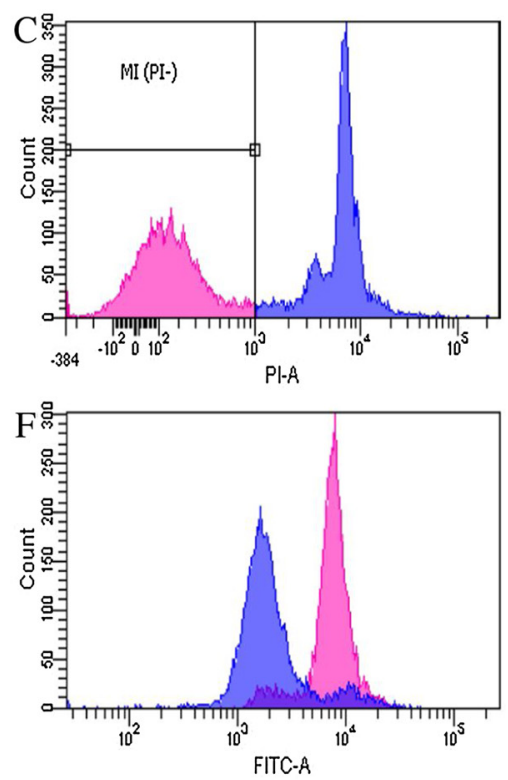

Fig. 1. Representative flow cytometry images of stallion cryopreserved semen ( $\{$ Control, A \& D ; containing coenzyme Q10 on the centrifugation $\{$ CE75; B \& E $\}$ or freezing \{FR100, C\& F $\}$ extenders), stained with propidium iodide fluorescence (PI, P4170, Sigma) and dihydrorhodamine 123 (DHR, D23806, Life Technologies). (A-C) Depict the percentiles of sperm with presenting plasma membrane integrity (pink colored, MI PI-, i.e., negative staining for PI). (D-F) Depict the assessment of fluorescence intensity (U.A.) for intracellular $\mathrm{H}_{2} \mathrm{O}_{2}$; Cells with damaged plasma membrane (i.e., PI + ) are stained in blue and cells with intact plasma membrane (PI-) are displayed in pink. (For interpretation of the references to colour in this figure legend, the reader is referred to the web version of this article).

incubation of semen samples in the presence of coenzyme Q10 preserved total and progressive sperm motility in men with normospermia (Lewin and Lavon, 1997) asthenozoospermia (Talevi et al., 2013).

Interesting that total and progressive sperm motility parameters were reduced after $30 \mathrm{~min}$ of incubation at $37^{\circ} \mathrm{C}$ for stallions classified as having bad semen freezing ability for the control group. However, coenzyme Q10 treated groups maintained these parameters during similar storage time. In the present study, addition of coenzyme Q10 promoted plasma membrane integrity for stallions classified as having bad semen freezing ability. This finding is consistent with the antioxidant properties of coenzyme Q10 inhibiting the formation of hydroperoxides and thus protecting the plasma membrane against oxidation (Turunen et al., 2004). It seems that there might be an association between higher percentage of cells with damaged membrane and reduced sperm motility. Despite inclusion of coenzyme Q10, sperm velocity was reduced across all concentrations in stallions classified as having bad semen freezing ability after $30 \mathrm{~min}$ of incubation. This decrease might be due to other cellular lesions not assessed by the methods employed in the present study.

Lipid peroxidation is determined by a sequence of reactions triggered by $\mathrm{OH}$, sequestering $\mathrm{H}^{+}$from the polyunsaturated fatty acids present in the phospholipid bilayer of the membranes, subsequently giving rise to alkoxyl (RO•) or peroxyl (ROO•) radicals, which tend to stabilize by withdrawing $\mathrm{H}^{+}$and an electron present in the adjacent phospholipids, generating a chain reaction and culminating in the disruption of the plasma membrane (Halliwell, 2006). The lipid peroxidation assessment demonstrated that addition to coenzyme Q10 before or after centrifugation decreased peroxidation when compared to the control. Interesting while the control group had higher lipid peroxidation, stallions classified as having good freezing ability appear to be able to restrain the peroxidation chain reaction since the plasma membrane integrity was not different when coenzyme Q10 was added to the extenders used during centrifugation or freezing. This suggests that the endogenous antioxidants, present in the seminal fluids of these animals, can stabilize the peroxyl radical efficiently. Conversely, stallions classified as having bad semen freezing ability responded well to the addition of coenzyme Q10. Previous studies with humans, bulls, and horses have shown that coenzyme Q10 alone or in combination with other molecules (e.g., $\alpha$-tocopherol, zinc, d-aspartate) resulted in reduced lipid peroxidation during cooled-storage or cryopreservation (Gualtieri et al., 2014; Nogueira, 2015; Talevi et al., 2013;Yousefian et al., 2014). Collectively, the authors suggested that coenzyme Q10 acts as an excellent tool in repairing plasma membrane $\alpha$-tocopherol polyunsaturated fatty acids.

Lipid peroxidation was a significant increased in both groups after $30 \mathrm{~min}$ of incubation at $37^{\circ} \mathrm{C}$. This finding may be explained by the greater exposure of sperm cells to free radicals during the $30 \mathrm{~min}$ of incubation when compared to the initial assessment. However, in the non-capacitated spermatozoa and plasma membrane assessments, only the control group of the stallions classified as having bad semen freezing ability presented a significant increase after $30 \mathrm{~min}$ of incubation.

Superoxide anion is generated primarily during the mitochondrial respiratory chain and is the precursor for most of ROS. This free radical reacts with $\mathrm{H}_{2} \mathrm{O}_{2}$ (Haber-Weiss' reaction) to form the hydroxyl radical, which is considered one of the most potent oxidizing agents, being able to cross membranes and react with molecules in the unsaturated lipids in cell membranes and DNA. Interesting that 
Control
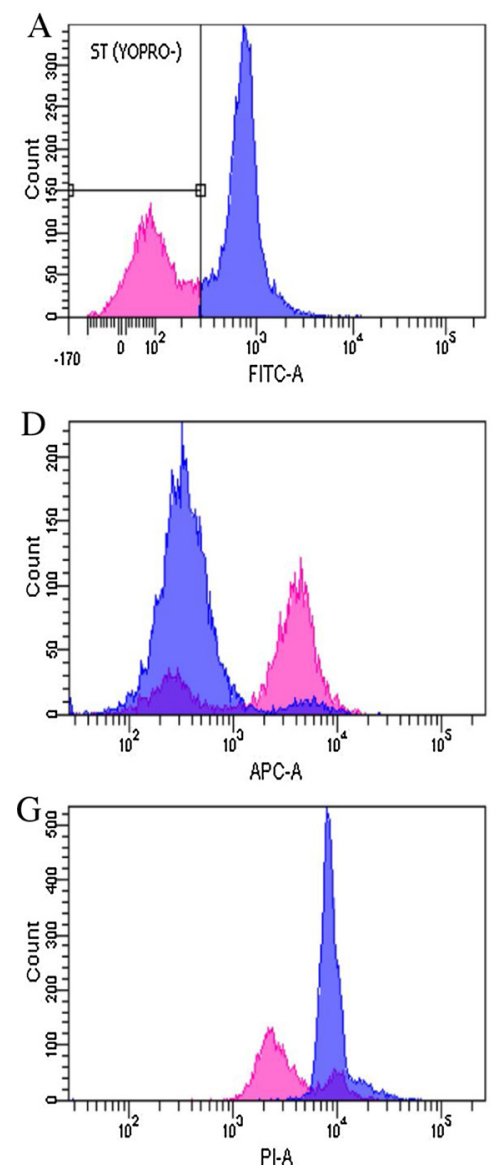

CE75
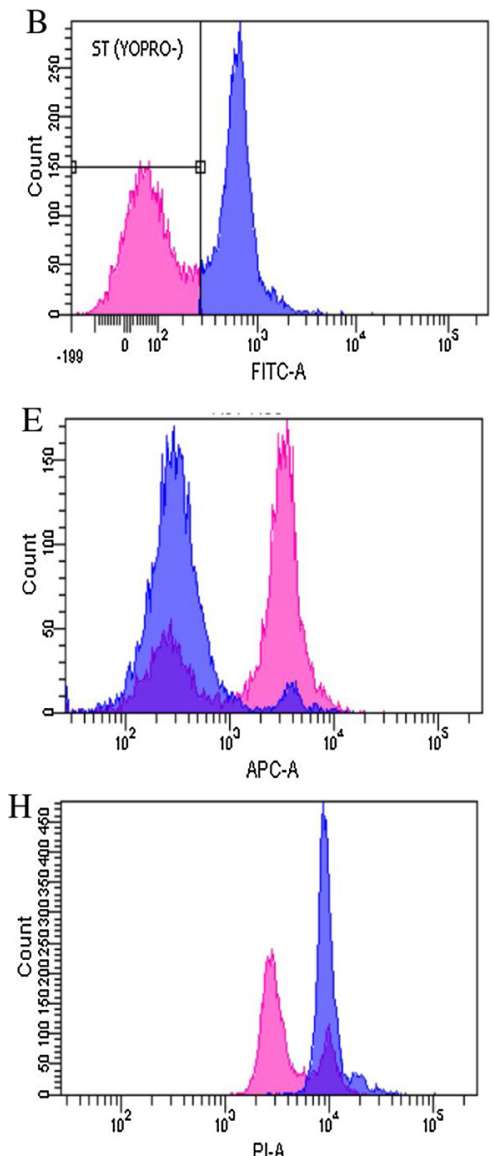

FR100
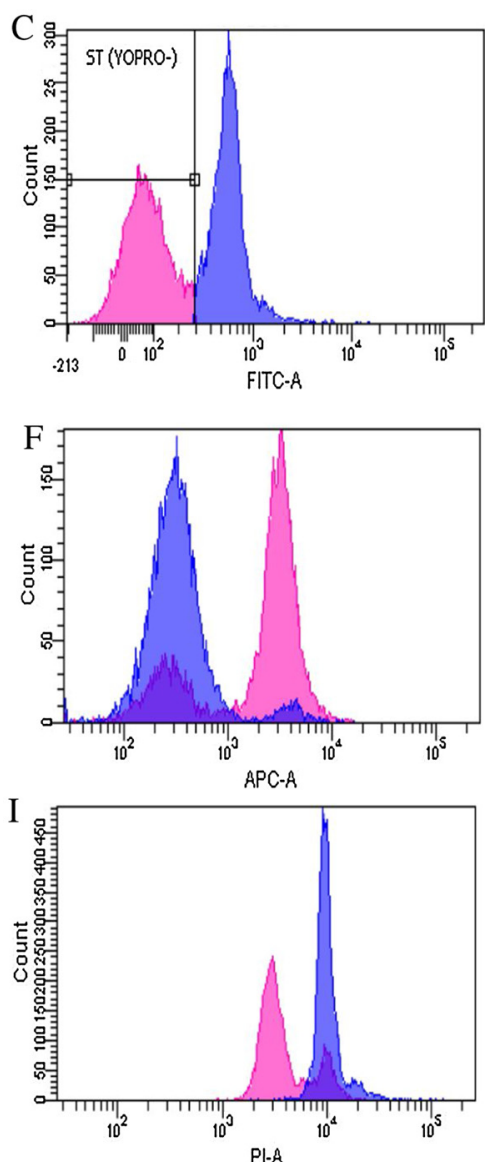

Fig. 2. Representative flow cytometry images of stallion cryopreserved semen ( Control, A, D \& G\}; containing coenzyme Q10 on the centrifugation \{CE75; B, E \& H\} or freezing $\left\{\right.$ FR100, C, F \& I\} extenders), stained with MitoStatus ${ }^{\circledR}$ Red (564,697 - BD Pharmigen) in association with Yopro ${ }^{\circledR}$ (Y3603 Life Technologies) and Dihydroethidium (D23107 Life Technologies). (A-C) represent sperm cells with stable plasma membrane (\% of ST, Yopro-), where blue colored populations represent sperm cells with destabilized plasma membrane (Yopro + ) and pink colored represent sperm cells with stabilized plasma membrane (Yopro-). (D-F) representative of the mitochondrial potential (MitoStatus Red) evaluated according to the fluorescence intensity (U.A.), where x-axis represent fluorescence intensity (APC). (G-I) Represent histograms used for assessment of superoxide anion based on fluorescence intensity (U.A.) in the mitochondrial sheath (Dihydroethidium). (For interpretation of the references to colour in this figure legend, the reader is referred to the web version of this article).

\section{Control}

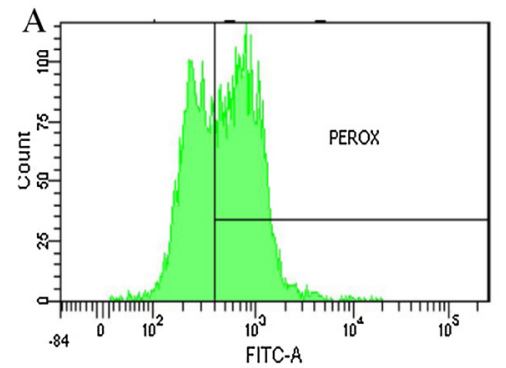

CE75

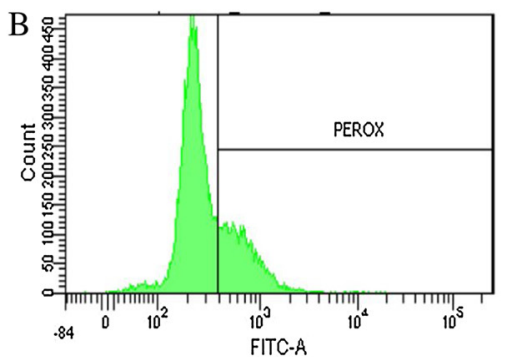

FR100

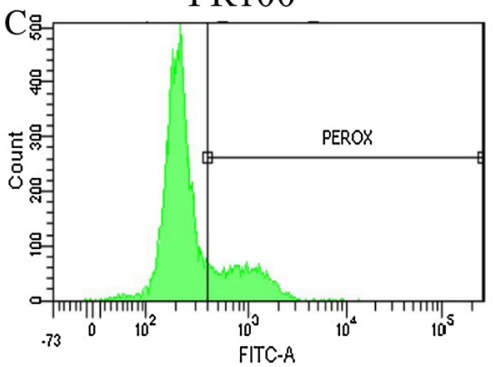

Fig. 3. Representative flow cytometry images of stallion cryopreserved semen (\{Control A\}, containing coenzyme Q10 on the centrifugation $\{$ CE75 B\} or freezing \{FR100 C \} extenders), stained with C11-BODYPY fluorescence (D-3861). Histograms A, B and C represent the percentile of cells with lipid peroxidation (\% of ST) for control, CE75 and FR100 groups, respectively.

addition of coenzyme Q10 did not affect $\mathrm{O}_{2}{ }^{-}$concentrations immediately after thawing and within 30 min. This might be explainable that coenzyme Q10 does not act affect directly the production of ROS, but it does act during the initiation stage phase of lipid peroxidation by neutralizing the lipid radicals after formation (Turunen et al., 2004).

Hydrogen peroxide $\left(\mathrm{H}_{2} \mathrm{O}_{2}\right)$ is formed from the dismutation of anion $\mathrm{O}_{2}{ }^{-}$and, because it is a chemically stable molecule, it is not 


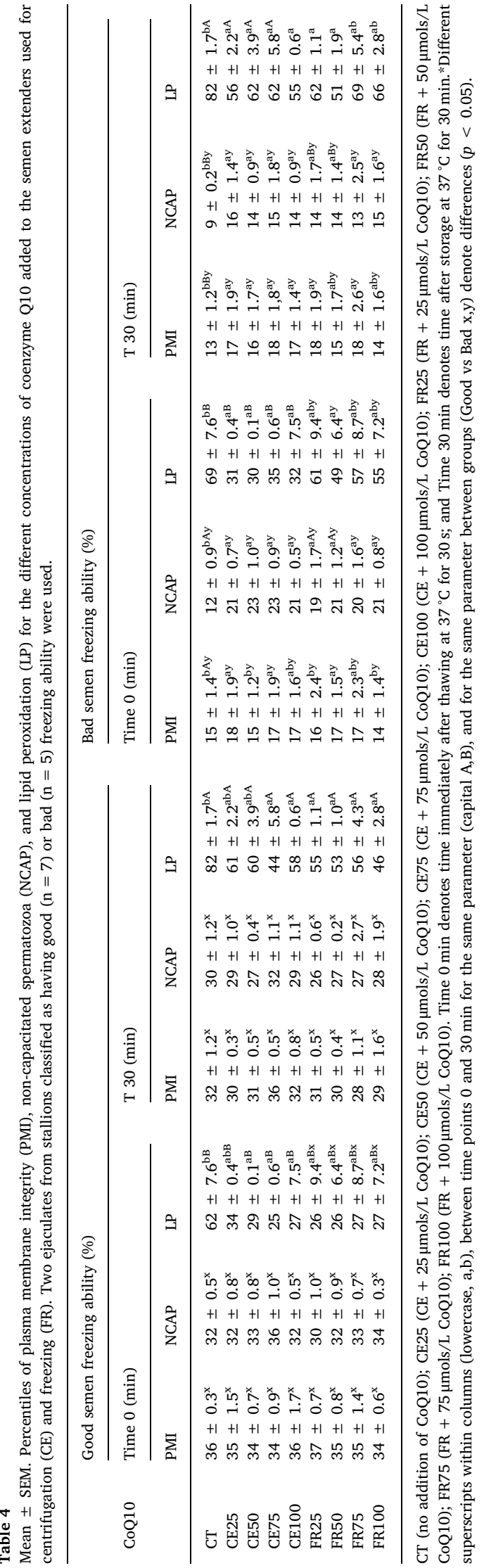




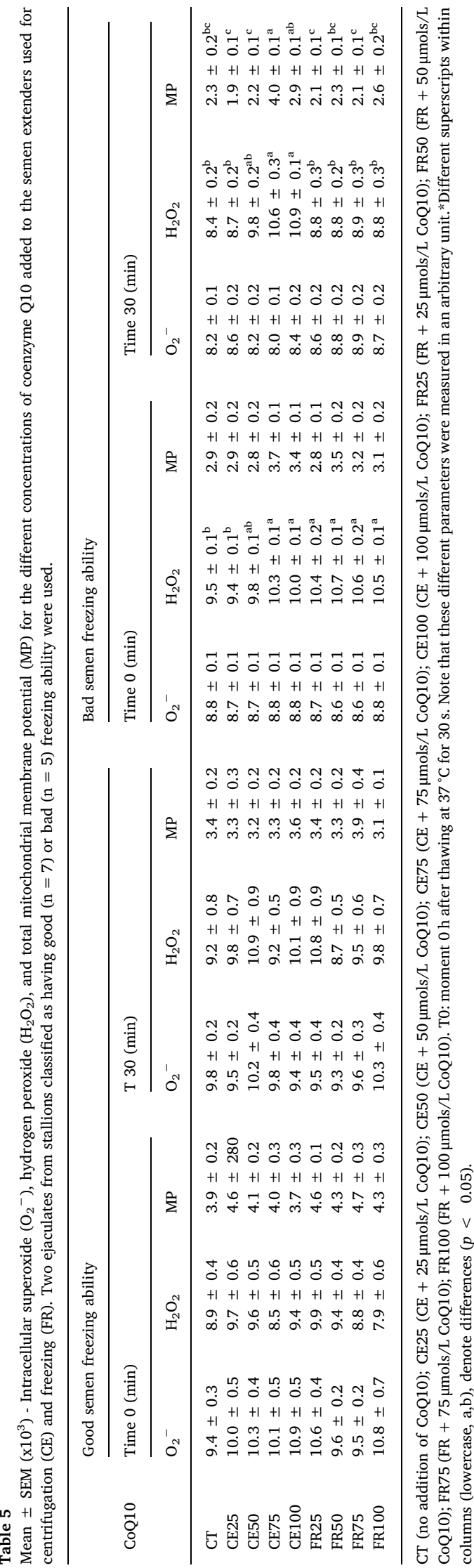


considered a free radical. However, hydrogen peroxide is present in high concentrations in the seminal plasma of stallions with good semen quality and is suggested to have a positive correlation with normal sperm metabolism (Gibb et al., 2014). The higher concentration of $\mathrm{H}_{2} \mathrm{O}_{2}$ in the treated groups (CE75 and CE100) in comparison to the control group, suggests that coenzyme Q10 promoted a greater protection of the metabolic activity for the spermatozoa of these groups in the animals classified as having bad semen freezing ability. Of interest, the most fertile stallions have higher concentrations of ROS than stallions with lower fertility and, consequently, have higher levels of oxidative stress (Gibb et al., 2014). These authors suggest that $\mathrm{H}_{2} \mathrm{O}_{2}$ production is simply a byproduct of high mitochondrial activity, showing a positive correlation between ROS levels and fertility rate in stallions. However, this is argued by another study showing a negative association between $\mathrm{H}_{2} \mathrm{O}_{2}$ concentrations, progressive motility, chromatin integrity, and fertility of cooled shipped stallion (Johannisson et al., 2014).

The ability of the sperm to produce ATP is indicated by mitochondrial activity and is positively correlated with kinetic parameters and sperm viability (Garner et al., 1997). Production of ROS can affect mitochondrial function by altering ATP synthesis, calcium homeostasis and transport of metabolites (Baumber et al., 2000). Stallions classified as having good and bad semen freezing ability were able to maintain mitochondrial activity irrespective of the addition of coenzyme Q10 immediately after thawing (T0). However, when the spermatozoa from stallions with bad freezing ability were submitted to metabolic stress (characterized by high production of ROSs and oxidative stress), for $30 \mathrm{~min}$ at $37^{\circ} \mathrm{C}$, only sperm cells treated with high concentrations (75 and $100 \mu \mathrm{mol} / \mathrm{L}$ ) of coenzyme Q10 through the centrifugation extender were able to maintain their metabolic activity. This result differs from that found by Baumber et al. (2005), using different concentrations (25 $\mu \mathrm{M}$ until $1 \mathrm{mM}$ ) of $\alpha$-tocopherol and Franco et al. (2013) using 0,5; 1,0; e $2,0 \mathrm{mM}$ of the same non-enzymatic antioxidants in the freezing extender for equine semen. After thawing, the samples were kept maintained at $37^{\circ} \mathrm{C}$ and the analyses performed at 0,60 and $120 \mathrm{~min}$. For both studies, did not maintain the mitochondrial activity of sperm cells. Possibly this difference occurred due to the methodology used, since, the animals were not classified as having bad or good semen freezing ability.

The presence of ROS, in moderate concentrations, is essential for the destabilization of the sperm membrane before capacitation. However, high concentrations of these elements can cause serious damage to the spermatozoa (Valença and Guerra, 2007). In the present study, addition of coenzyme Q10 promoted superior plasma membrane stability to the sperm cells from stallions classified as having bad freezing ability when compared to the control group, demonstrating the inhibitory potential of the coenzyme to early capacitation. These findings resemble those found in bovine that the inhibition of sperm capacitation after addition of superoxide dismutase to semen did not occur after the addition of the superoxide anion (O'Flaherty et al., 1999).

There were no differences in time for mitochondrial potential, intracellular hydrogen peroxide (H2O2) and intracellular superperoxide (O2-) production analysis on either group of stallions. Despite the mitochondria being the most sensitive sperm structure to the cryopreservation process (Ortega et al., 2009), the 30 -min incubation at $37^{\circ} \mathrm{C}$ was probably not sufficient to exhaust energy supply provided by the post-thaw diluent, thus maintaining its accelerated metabolism and consequent the production of free radicals. It is also possible that the methods or number of animals used in the present study were not able to detect subtle differences between groups and treatments. While we started with a group of twenty stallions, only twelve stallions fitted the inclusion criteria.

The results of this study demonstrated that coenzyme Q10 was most effective when it was added to the extender used for centrifugation rather than the freezing extender. Although the centrifugation performed in the freezing process may be harmful and result in mechanical damage to the plasma membrane (Barrier-Battut et al., 2013), the addition of coenzyme Q10 before centrifugation appears to be able to compensate for the antioxidant deficiency in stallions with bad semen freezing ability. At centrifugation spermatozoon is at the peak of its energy metabolism, thus facilitating the absorption of substances and ionic exchanges (Squires et al., 1999). Antioxidant supplementation associated with seminal plasma extraction, and consequently byproducts of sperm metabolism (e.g., ROS), increases sperm viability during the cryopreservation process (Barrier-Battut et al., 2013).

Since coenzyme Q10 is liposoluble, it acts directly in the polyunsaturated lipid chains present in the plasma membrane. Possibly, the lipid composition present in the egg yolk, an essential component of the freezing extender, competes with the sperm cells in the use of coenzyme Q10, making its availability more limited to the sperm cells. This hypothesis appears to be supported by a study involving semen cryopreserving in bucks (Khalifa et al., 2008). These authors obtained the best results using 5 mM of non-enzymatic antioxidant butylated hydroxytoluene in extender containing egg yolk and $0.3 \mathrm{mM}$ of the same antioxidant in egg yolk free extender. According to the authors, when the extender contains egg yolk, it is necessary to increase the antioxidant concentration as a way of compensating losses by the interaction between the antioxidant and the polyunsaturated fatty acids present in the lipid components of the yolk.

In conclusion, sperm motility parameters were inferior for stallions classified as having bad semen freezing ability. There were no effects of coenzyme Q10 concentration or time for all parameters evaluated in the group with good semen freezing when compared with the control extender. Stallions with bad semen freezing ability had improved sperm parameters for samples processed with extenders containing coenzyme Q10, except for the $\mathrm{O}_{2}{ }^{-}$concentration and mitochondrial potential (T0) in which there was no difference between the groups.It appears that $75 \mu \mathrm{mols} / \mathrm{L}$ is the optimal dose of coenzyme Q10, particularly, when added to the centrifugation extender.

\section{Conflict of interest}

None. 


\section{Authors' contributions}

JAM, BRSB, VFCS collected and cryopreserved the semen and performed the post-thaw sperm motility analyses. JAM and CPFDA performed the flow cytometry analyses. JADA, MAA, FOP designed the study. JAM, JADA, IFC analyzed the data and wrote the manuscript. All authors reviewed the final manuscript.

\section{Acknowledgement}

The CNPq is acknowledged for providing stipends for the first author and Grant nos. 2015/25638-3 from the São Paulo Research Foundation (FAPESP) is acknowledged for funding the study. Botupharma (Botucatu, Sao Paulo, Brazil) kindly donated the extenders used in the study.

\section{References}

Agarwal, A., Majzoub, A., 2017. Role of antioxidants in assisted reproductive techniques. World J. Mens Health 35, 77-93.

Alleva, R., Scararmucci, A., Mantero, F., Bompadre, S., Leoni, L., Littarru, G.P., 1997. The protective role of ubiquinol-10 against formation of lipid Hydroperoxides in human seminal fluid. Mol. Aspects Med. 18 (Suppl), 221-228.

Andrade, E.R., Melo-Sterza, F.A., Seneda, M.M., Alfieri, A.A., 2010. Consequences of the production of reactive oxygen species in reproduction and main antioxidant mechanisms. Rev. Bras. de Reprodução Anim. 34, 79-85.

Barrier-Battut, I., Bonnet, C., Giraudo, A., Dubois, C., Caillaud, M., Vidament, M., 2013. Removal of seminal plasma enhances membrane stability on fresh and cooled stallion spermatozoa. Reprod. Domest. Anim. 48, 64-71.

Baumber, J., Ball, B.A., Gravance, C.G., Medina, V., Davies-Morel, M.C.G., 2000. The effect of reactive oxygen species on equine sperm motility, viability, acrosomal integrity, mitochondrial membrane potential, and membrane lipid peroxidation. J Androl. 21 (6), 895-902.

Baumber, J., Ball, B.A., Linfor, J.J., 2005. Assessment of cryopreservation of equine spermatozoa in the presence of enzyme scavengers and antioxidants. Am. J. Vet. Res. 57, 772-779.

Bentinger, M., Brismar, K., Dallner, G., 2007. The antioxidant role of coenzyme Q. Mitochondrion 7, 41-50.

Brito, L.F.C., 2007. Evaluation of stallion sperm morphology. Clin. Tech. Equine Pract. 6, 249-264.

Carocho, M., Ferreira, I.C.F.R., 2013. A review on antioxidants, prooxidants, and related controversy: natural and synthetic compounds, screening and analysis methodologies and future perspectives. Food Chem. Toxic. 51, 15-25.

Franco, J.S.V., Chaveiro, A., Gois, A., Silva, F.M., 2013. Effects of $\alpha$-tocopherol and ascorbic acid on equine semen quality after cryopreservation. J. Equine Vet. Sci. 33, 787-793.

García, B.M., Fernández, L.G., Ferrusola, C.O., Rodríguez, A.M., Bolaños, J.M.G., Martinez, H.R., Tapia, J.A., Morcuende, D., Peña, F.J., 2011. Fatty acids and plasmalogens of the phospholipids of the sperm membranes and their relation with the post-thaw quality of stallion spermatozoa. Theriogenology 75, 811-818.

Garner, D.L., Thomas, C.A., Joerg, H.W., Dejanertte, J.M., Marshall, C.E., 1997. Fluorometric assessments of mitochondrial function and viability in cryopreserved bovine spermatozoa. Biol. Reprod. 57, 1401-1406.

Gibb, Z., Aitken, R.J., 2016. The impact of sperm metabolism during in vitro storage: the stallion as a model. BioMed Res. Int. 8.

Gibb, Z., Lambourne, S.R., Aitken, R.J., 2014. The paradoxical relationship between stallion fertility and oxidative stress. Biol. Reprod. 91, 1-10.

Granollers, G.L., Varea, R.L., Vizcaíno, M.A.C., Monqaut, A., Macazzaga, M.B., 2011. Effect of treatment with vitamins, L-carnitine and coenzyme Q10 on the vacuolization and sperm fragmentation rate in fertilizations patients in vitro. Revista Internacional de Andrología 9, 154-159.

Gualtieri, R., Barbato, V., Fiorentino, I., Braun, S., Rizos, D., Longobardi, S., Talevi, R., 2014. Treatment with zinc, d-aspartate, and coenzyme Q10 protects bull sperm against damage and improves their ability to support embryo development. Theriogenology 82, 592-598.

Guasti, P.N., Freitas-Dell'aqua, C.P., Maziero, R.R.D., Monteiro, G.A., Hartwig, F.P., Lisboa, F.P., Papa, P.M., Papa, F.O., 2012. Lipid peroxidation and generation of hydrogen peroxide from subfertile stallion spermatozoa during storage at refrigeration temperature. 39th Annual Conference of the IETS, 2012, Hannover. Reprod Fertil Dev 25 20-20.

Gvozdjakova, A., Kucharska, J., Dubravicky, J., Mojto, V., Singh, R., 2015. Coenzyme Q10, $\alpha$-tocopherol, and oxidative stress could be important metabolic biomarkers of male infertility. Dis. Markers, 27941.

Gvozdjakova, A., Lipkova, J., Bartolcicova, B., Dubravicky, J., Vorakova, M., Cernakova, I., Singh, R.B., 2013. Importance of the assessment of coenzyme Q10, alphatocopherol and oxidative stress for the diagnosis and therapy of infertility in men. Bratisl. Med. J. 114, 607-609.

Halliwell, B., 2006. Oxidative stress and neurodegeneration. Where are we now? J. Neurochem. 97, 1634-1658.

Hartwig, F.P., Lisboa, F.P., Hartwig, F.P., Monteiro, G.A., Maziero, R.R., Freitas-Dell'aqua, C.P., Alvarenga, M.A., Papa, F.O., Dell'aqua Jr, J.A., 2014. Use of cholesterol-loaded cyclodextrin: an alternative for bad cooler stallions. Theriogenology 81, 340-346.

Johannisson, A., Lundgren, A., Humblot, P., Morrell, J.M., 2014. Naturally and stimulated levels of reactive oxygen species in cooled stallion semen destined for artificial insemination. Animal 10, 1-9.

Khalifa, T.A., Lymberopoulos, A.G., El-Saidy, B.E., 2008. Testing usability of butylated hydroxytoluene in conservation of goat semen. Reprod. Domest. Anim. 43, 525-530.

Lenzi, A., Gandini, L., Lombardo, F., Picardo, M., Maresca, V., Panfili, E., Tramer, F., Boitani, C., Dondero, F., 2002. Polyunsaturated fatty acids of germ cell membranes, glutathione, and glutathione-dependent enzyme-PHGPx: from basic to clinic. Contraception 65, 301-304.

Lewin, A., Lavon, H., 1997. The effect of coenzyme Q10 on sperm motility and function. Mol. Aspects Med. 18, $213-219$.

Luz, H.K.M., Wanderley, L.S., Faustino, L.R., Silva, C.M.G., Figueiredo, J.R., Rodrigues, A.P.R., 2011. Role of antioxidant agents in cryopreservation of germ cells and embryos. Acta Scientiae Veterinariae 39, 956-969.

Martin, J.C., Klug, E., Gunzel, A.R., 1979. Centrifugation of stallion semen and its storage in large volume straws. J. Reprod. Fertil. Suppl. 27 , 47-51.

Maziero, R.R.D., 2010. Comparison between different freezing rates and semen storage volumes on sperm viability in stallions of high and low freeze. Dissertação Mestrado. Faculdade de Medicina Veterinária e Zootecnia, Universidade Estadual Paulista, Botucatu.

Moore, I., Squires, E.L., Graham, J.K., 2005. Effect of seminal plasma on cryopreservation of equine spermatozoa. Theriogenology 63, $2372-2381$.

Morillo-Rodríguez, A., Macías-García, B., Tapia, J.A., Ortega-Ferrusola, C., Peña, F.J., 2012. Consequences of butylated hydroxytoluene in the freezing extender on post-thaw characteristics of stallion spermatozoa in vitro. Andrologia 44, 688-695.

Nogueira, B.G., 2015. Coenzima Q10 e $\alpha$-Tocoferol Previnem a Lipoperoxidação do sêmen equino refrigerado. Dissertação (Mestrado) - Faculdade de Medicina Veterinária e Zootecnia da Fundação Universidade Federal de Mato Grosso do Sul, Mato Grosso do Sul.

O'Flaherty, C.M., Beorlegui, N.B., Beconi, M.T., 1999. Reactive oxygen species requirements for bovine sperm capacitation and acrosome reaction. Theriogenology 52, 289-301.

Ortega, F.C., González, F.L., Morrell, J.M., Salazar, S.C., Macías, G.B., Rodríguez-Martinez, H., Tapia, J.A., Peña, F.J., 2009. Lipid peroxidation, assessed with BODIPYC11, increases after cryopreservation of stallion spermatozoa, is stallion dependent and is related to apoptotic-like changes. Reproduction 138 (1), 55-63.

Papa, F.O., Melo, C.M., Fioratti, E.G., Dell'Aqua Jr, J.A., Zahn, F.S., Alvarenga, M.A., 2008. Freezing of stallion epididymal sperm. Anim. Reprod. Sci 107, 293-301.

Parrish, J.J., Susko-Parrish, J., Winer, M.A., First, N.L., 1988. Capacitation of bovine sperm by heparin. Biol. Reprod. 38, 1171-1180.

Patel, S.R., Sigman, M., 2008. Antioxidant therapy in male infertility. Urol. Clin. North Am. 35, 319-330. 
Pisoschi, A.M., Pop, A., 2015. The role of antioxidants in the chemistry of oxidative stress: a review. Eur. J. Med. Chem. 97, 55-74.

Rodríguez, A.M., Ferrusola, C.O., García, B.M., Morrell, J.M., Martínez, H.R., Tapia, J.A., Peña, F.J., 2011. Freezing stallion semen with the new Cáceres extender improves post-thaw sperm quality and diminishes stallion-to-stallion variability. Anim. Reprod. Sci. 127, $78-83$.

Seifi-Jamadi, A., Kohram, H., Shahneh, A.Z., Ansari, M., Macías-García, B., 2016a. Quercetin ameliorate motility in frozen-thawed Turkmen stallions sperm. J. Equine Vet. Sci. 45, 73-77.

Seifi-Jamadi, A., Kohrama, H., Shahnea, A.Z., Dehghanizadeha, P., Ahmadc, E., 2016b. Effect of various concentrations of butylated hydroxyanisole and butylated hydroxytoluene on freezing capacity of Turkman stallion sperm. Anim. Reprod. Sci. 170, 108-113.

Sieme, H., Harrison, R.A.P., Petrunkina, A.M., 2008. Cryobiological determinants of frozen semen quality, with special reference to stallion. Anim. Reprod. Sci. 107, $276-292$.

Squires, E.L., Pickett, B.W., Graham, J.K., Vanderwall, D.K., Mccue, P.M., Bruemmer, J.E., 1999. Cooled and frozen stallion semen. Anim. Reprod. Biotechnol. Lab. 9, $01-38$.

Squires, R.L., Keith, S.L., Graham, J.K., 2004. Evaluation of alternative cryoprotectants for preserving stallion spermatozoa. Theriogenology 62, $1056-1065$.

Talevi, R., Barbato, V., Fiorentino, I., Braun, S., Longobardi, S., Gualtieri, R., 2013. Protective effects of in vitro treatment with zinc, d-aspartate and coenzyme Q10 on human sperm motility, lipid peroxidation and DNA fragmentation. Reprod. Biol. Endocrinol. 11, 1-10.

Turunen, M., Olsson, J., Dallner, G., 2004. Metabolism, and function of coenzyme Q. Biochim. Biophys. Acta 1660, 171-199.

Valença, R.M.B., Guerra, M.M.P., 2007. Espécies reativas ao oxigênio (ROS) e a utilização de antioxidantes na criopreservação do sêmen suíno. Rev. Bras. Reprod, Anim. 31, 47-53.

Varner, D.D., Gibb, Z., Aitken, R.J., 2014. Stallion fertility: a focus on the spermatozoon. Equine Vet. J. 47, 16-24.

Vidament, M., 2005. French field results (1985-2005) on factors affecting fertility of frozen stallion semen. Anim. Reprod. Sci. 89, 115-136.

Yousefian, I., Zare-Shahneh, A., Mahdi Zhandi, M., 2014. The effect of coenzyme Q10 and $\alpha$-tocopherol in skim milk-based extender for preservation of Caspian stallion. J Equine Vet Sci. 34, 949-954. 